\title{
The circumsolar dust complex and solar magnetic field
}

\author{
Alexander Krivov ${ }^{1,2,3}$, Ingrid Mann ${ }^{1}$, and Hiroshi Kimura ${ }^{1}$ \\ ${ }^{1}$ Max-Planck-Institut für Aeronomie, Katlenburg-Lindau, Germany \\ ${ }^{2}$ Space Research Center, Warsaw, Poland \\ ${ }^{3}$ On leave from Astronomical Institute, St. Petersburg University, Russia
}

(Received October 7, 1997; Revised January 15, 1998; Accepted February 12, 1998)

\begin{abstract}
New model calculations for the dynamical evolution of dust particles at several solar radii around the Sun are presented. We choose a fractal aggregate consisting of either silicate or carbon as a representative of dielectric and absorbing fluffy particles. We take into account a large array of forces and effects acting on the dust particlessolar gravity, direct solar radiation pressure, Poynting-Robertson effect, sublimation, and the Lorentz force, with a special emphasis given to the latter. The Lorentz force was computed on the base of modeled grain's charges and a model of the actual solar magnetic field from 1976 to 1996. We have investigated the dynamics of individual grains, obtained radial and vertical density profiles of different-sized particles, and used the computed dust density distributions to calculate the expected $F$-corona brightness during the periods of weak and strong magnetic field. We have found that the solar magnetic field and its variations do not affect the dynamics and spatial distribution of carbon aggregates, which are thought to produce the peak features of the near-infrared $F$-corona brightness that were sometimes observed. On the other hand, the variations of the solar magnetic field may alter the latitudinal distribution of silicate aggregates. However, the effect is not strong enough to account for the observed temporal variations in the brightness. Thus we can rule out the correlation between the appearance or disappearance of a peak feature and the solar activity cycle.
\end{abstract}

\section{Statement of the Problem}

As the eventual destination for most of the interplanetary dust grains (IDPs) by virtue of their Poynting-Robertson evolution and a possible birthplace for $\beta$-meteoroids, the nearsolar dust complex plays an important role in the maintenance and evolution of the zodiacal cloud. The existence of the circumsolar dust ring was theoretically predicted by Belton (1966, 1967), who suggested the formation of the ring as a result of increasing radiation pressure forces acting on dust particles, as they reduce in size due to partial sublimation near the Sun. Successful observations of the $F$-corona brightness enhancement near $4 R_{\odot}$ first made by MacQueen (1968) and Peterson $(1967,1969)$ have supported this prediction. Since that time, many authors have tried to fit the observational data with their models. By considering spherical silicate and carbon particles and their dynamical behavior near the Sun, Mukai and Yamamoto (1979) constructed a model of the dust ring and explained the observed feature of the near-infrared brightness by thermal emission of the carbon particles superimposed on the sunlight scattered by silicate grains. Using the idea of a superposition of scattered light and thermal emission components, Mann (1992) pointed out that the formation of a feature in the near-infrared brightness does not necessarily require the presence of a dust ring. Mann showed that a peak feature can be explained as a geometric effect that occurs from the sharp decrease of the thermal emission brightness at the point where the line of sight crosses the be-

Copy right (C) The Society of Geomagnetism and Earth, Planetary and Space Sciences (SGEPSS); The Seismological Society of Japan; The Volcanological Society of Japan; The Geodetic Society of Japan; The Japanese Society for Planetary Sciences. ginning of the dust-free zone. As was found later by Kimura et al. (1998), the appearance of a feature strongly depends on the thermal emission brightness at the wavelength of observations, which, in turn, is highly sensitive to the material composition of near-solar dust. What further complicates the problem is that the observations made during different solar eclipses give different results. A detailed analysis of the $F$-corona brightness observations (Kimura and Mann, $1998 \mathrm{~b}$, this issue) made it clear that in nearly half the cases the $F$-corona brightness observations showed the presence of the peak feature, whereas in the others the peak feature was not observed. At present there is no unanimity about the explanation of this fact.

This paper presents model calculations of dynamics and distribution of charged dust near the Sun. As distinct to the previous studies, we give, for the first time, a quantitative consideration of the influence of the Lorentz force on the near-solar dust. Its importance for the evolution of dust in the close vicinities of the Sun was noted by Rusk (1988) and Mann and Kimura (1997). In addition, Isobe and Kumar (1993) have suggested that variations of the Lorentz force due to solar activity cycle could lead to the temporal variations in the circumsolar dust complex, and therefore might explain the detections/nondetections of the peak feature mentioned above. We will not provide a further discussion of the different observational results which is given in Kimura and Mann (1998b, this issue). However, we use our model to check, from the point of view of the dust dynamics, whether the variation of the solar magnetic field and the resulting variation of the Lorentz forces can explain a variability of the $F$-corona brightness. 


\section{Model Description}

In our modeling of the circumsolar dust complex, the following forces were taken into account: solar gravity, direct radiation pressure, Poynting-Robertson force, sublimation, and Lorentz force. Because of the complexity of the problem, we neglect a number of other forces and effects which, in principle, can affect the results and have to be examined in further studies. Most notably, we do not take into account the pseudo Poynting-Robertson drag due to interactions of dust grains with solar wind particles which is able to amplify the Poynting-Robertson effect by 30 to $70 \%$ (Banaszkiewicz et al., 1994; Shestakova and Tambovtseva, 1995). Two chemical compositions of dust particles were considered: silicate and carbon, which represented dielectric and absorbing grains, respectively. We describe the grains as ballistic particle-cluster aggregates (BPCAs, see Mukai et al., 1992). This model has been used before for calculations of temperature profiles of near-solar dust (Mann et al., 1994). Note that using compact spherical particles instead of BPCAs would lead to some quantitative corrections, particularly decreasing the charge to mass ratio of grains and hence reducing the effect of the Lorentz force, but it would not change the results qualitatively.

The computation of gravitational and radiation pressure forces and sublimation rates for BPCA grains was based on formulas and algorithms of Kimura et al. (1997). To calculate the electromagnetic force, we had to model grain charges and solar magnetic field strengths. The former for fractal aggregates were evaluated taking full account for sticking and penetration of solar wind particles into dust grains, secondary electron emission, photoelectron emission, and thermionic emission, that resulted in typical electrostatic potentials of +3 to $+9 \mathrm{~V}$, using the technique similar to that described in Kimura and Mann (1998a). Contrary to predictions of Isobe and Kumar (1993), our calculations do not show any sizeable dependence of the charges on the solar activity cycle.

To determine the magnetic field, we made use of the Potential Field-Source Surface Model of Hoeksema and Scherrer (1986) with harmonic coefficients of Wilcox Solar Observatory, representing actual solar field for 1976 through 1996. We then compared characteristic strengths of the magnetic field to sunspot numbers. It turned out that the sunspot numbers did not closely relate to the global magnetic field strength. For example, in 1982.5 and 1991.0 (near the dates when high-quality eclipse observations were made) the magnetic field was respectively strong and weak, whereas sunspot numbers were respectively moderate and high.

For the model described above, the equations of motion and equation of sublimation rate were integrated simultaneously-first for a moderate number of trajectories to study the typical features of dust grains' evolution and then for a larger number of orbits to obtain spatial distributions of near-solar grains.

\section{Single-Particle Dynamics}

The evolution of dust grains in the orbital plane was found to be almost unaffected by the Lorentz force. For carbon particles, sublimation begins at $3.8-4.2 R_{\odot}$ and continues until a radius of $\approx 2.4 \mu \mathrm{m}$ is reached (that corresponds to the radiation pressure to gravity ratio $\beta \approx 1$ ). Then the particles, keeping a nearly constant size, exhibit a number of radial oscillations. Finally they are ejected out of the solar vicinities in hyperbolic trajectories, becoming $\beta$-meteoroids. Silicate particles start to sublimate at $2.2-2.5 R_{\odot}$ and rapidly disappear there.

In contrast, the evolution of grains' orbital planes is dictated by the Lorentz force but, like the "radial" dynamics, it is distinct for carbon and silicate particles. Even for the smallest carbon grains which are not swept out by radiation pressure $(2.4 \mu \mathrm{m})$, the maximum inclinations are comparable to the median values for the inclination distribution according to different models of the interplanetary dust cloud $(\approx 15$ to $20^{\circ}$ ). Hence the Lorentz force has little effect on these particles and would not cause a significant modification of the overall spatial distribution of dust near the Sun. Silicate particles behave differently. Bigger grains ( $\gtrsim 2 \mu \mathrm{m}$ and $\gtrsim 1 \mu \mathrm{m}$ for periods of quiet and active Sun, respectively) remain essentially unaffected by the Lorentz force, but the smaller grains may develop large inclinations. Grains of $1 \mu \mathrm{m}$ in size may reach polar orbits, if the magnetic field is strong enough. The dynamics of submicrometer-sized silicate particles is extremely sensitive to the Lorentz force. Even near the solar activity minima, the inclinations are distributed almost uniformly between 0 and $180^{\circ}$, so that the retrograde motions are as typical as prograde ones.

\section{Spatial Distribution of Dust}

The next step of the study was to estimate the number density distributions of near-solar dust, in order to check possible temporal variations caused by the Lorentz force. Since elucidation of the actual spatial distribution of dust is beyond the scope of this paper, we assumed a relatively simple conventional model of the dust cloud, rather than a realistic sophisticated one. Different-sized dust grains were launched at different time instants from initially uninclined, circular orbits at $5 R_{\odot}$ from the Sun. We performed numerical integrations of 540 individual trajectories to get more than 200,000 instantaneous positions of particles, and then derived spatial distributions of the grains by counting the occurrences of grains in various spatial bins (see Krivov and Hamilton, 1997, for more details about this technique). The absolute number densities were then calculated from the relative ones by means of IDP flux model at $1 \mathrm{AU}$ of Grün et al. (1985), assuming that a half of the flux consists of the silicate particles.

In such a way, we first constructed radial distributions of different-sized silicate and carbon BPCA grains near the Sun in the periods of relatively strong and weak solar magnetic field (1983 and 1991, respectively). In both cases, we saw an excess of carbon particles at $3.5-3.7 R_{\odot}$ and a gently sloping distribution of silicate particles. No differences between the 1983 and 1991 distributions of circumsolar dust were found.

Second, we obtained vertical (latitudinal) profiles of the same grains for the same two periods. The distributions of carbon grains for both dates (1983 and 1991) did not show any differences, nor did those of large $(\gtrless 5 \mu \mathrm{m})$ and small $(\leqq 0.5 \mu \mathrm{m})$ silicate particles. However, the distributions of medium-sized silicate dust were not similar: the 1991 profile proved to be more confined to the ecliptic plane than the 1983 one (Fig. 1). This was the only effect of the magnetic field on 


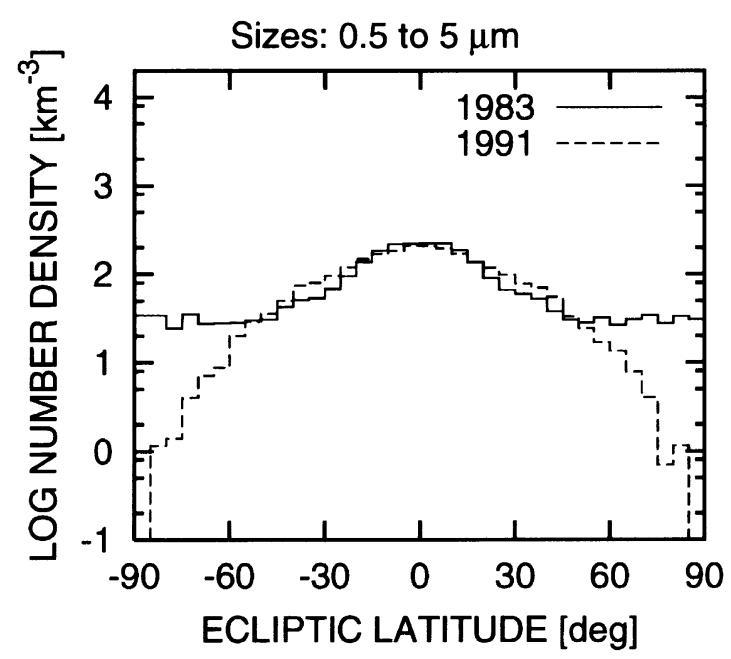

Fig. 1. Modeled latitudinal distributions of medium-sized silicate BPCA grains near the Sun in the periods of strong (1983) and weak (1991) solar magnetic field.

the near-solar dust complex that resulted from our modeling.

\section{Discussion}

As discussed in the Introduction, Isobe and Kumar (1993) expressed the idea that the apparent variability of the $F$ corona brightness might be explained by changes in the Lorentz force acting on the charged near-solar dust. They argue that both grains' charges and magnetic field strength depend on the solar activity phase and expect that the Lorentz force would displace the dust toward or outward the Sun. Our modeling does not show a strong response of the charges to the solar activity cycle, nor does it confirm the radial movements of the dust material due to the Lorentz force. Yet it does not reject a general idea that the Lorentz force may influence the near-solar dust complex. Let us look at the problem from another viewpoint.

The hump feature in the $F$-corona brightness is believed to be caused by thermal emission of absorbing grains (carbon ones in our model). But such grains necessarily possess high radiation pressure to gravity ratios, $\beta$, so that only large, micrometer-sized absorbing particles can be present in the near-solar region-smaller ones are swept out by radiation pressure. However, the grains of that size are too large to be appreciably affected by the Lorentz force, as follows from simple force estimates and was confirmed by our modeling. Hence the peak feature should not correlate with solar activity variations.

There may be another way to link observational facts to the Lorentz force and solar activity cycle. The peak feature is observed on a background brightness produced by light scattering on dielectric grains. Such grains, even submicrometer-sized ones, have $\beta$ ratios smaller than unity and must be present. As shown above, such particles are subject to the Lorentz force and their distribution does depend on the strength of the solar magnetic field. This suggests an alternative idea: the observability of the peak feature may depend on the amount of dielectric particles which produce a smooth brightness profile as opposed to the feature-like pro- file produced by the absorbing particles. When the magnetic field is strong enough, the dielectric particles are scattered over a wide range of latitudes, consequently only producing a weak background brightness close to the equatorial plane and allowing for the feature produced by the absorbing grains to be seen against the background radiation coming from dielectric particles. When the field gets weaker, the dielectric grains concentrate toward the ecliptic plane, and the peak feature would disappear at the stronger background. We have made model calculations of the $F$-corona brightness to see whether the variations in latitudinal distribution of the silicate dust (Fig. 1) are strong enough to produce observational consequences. The result is negative. It can be explained by the fact that the main contribution to the continuum is made by relatively large line-of-sight silicate particles located closer to the Earth (the effect of scattering angles, see van de Hulst, 1947). Thus we can rule out this hypothesis as well.

Conceivably the facts in question could be explained by individual events that might change mean chemical composition or the amount of dust material near the Sun. As was discussed by Mann (1996), some of the findings about the near solar dust cloud point to the existence of an out-ofecliptic dust component. Such a component, if produced by long period comets, may have a different collisional evolution than the dust cloud concentrated to the ecliptic (see Ishimoto, 1998, this issue). It may also cause a time variation in the composition of the near solar dust cloud. At this point, further observational results about comets in the inner solar system as well as about the temporal variation of the $F$-corona brightness may yield a better understanding in the future.

\section{Summary}

1. We perform new calculations for dynamics of nearsolar dust grains, using a fractal aggregate model consisting of silicate and carbon as a representative of dielectric and absorbing fluffy particles, taking into account solar gravity, direct solar radiation pressure, Poynting-Robertson effect, sublimation, and the Lorentz force, with a special emphasis given to the latter.

2. Using the model above, we have investigated the dynamics of individual grains, and discussed consequences for the dust number density distribution and the expected $F$ corona brightness during the periods of weak and strong magnetic field.

3. Our calculations strongly suggest that the appearance or disappearance of a peak feature in the $F$-corona brightness near $4 R_{\odot}$ is not connected to a solar activity cycle. We conclude that the presence of a peak feature during 19661967 and 1983 solar eclipses and its absence in other periods should be due to different effects.

Acknowledgments. The authors thank Dr. J. Todd Hoeksema for kind help in using the solar magnetic field data, Prof. H. Fahr and Dr. H. Ishimoto for careful reviews, and Prof. J. F. McKenzie for reading the manuscript and correcting the style. This research was supported by the Bundesministerium für Bildung, Wissenschaft, Forschung und Technologie (BMBF). The work of A.K. was partially supported by the RBRF grant 96-02-19638. Much of the work was done during the stays of A.K. at MPI für Aeronomie (MPAE) and at Space Research Center (SRC, Warsaw); the stay at SRC 
was funded by grants of Józef Mianowski Fund and Foundation for Polish Science (Poland) and State Committee for Higher Education (Russia). This study was presented at the IAU General Assembly (Kyoto, 18-30 August 1997) and Zodiacal Cloud Sciences Workshop in Kobe (1-3 September 1997); participation of A.K. in these conferences was supported by LOC of IAU GA and MPAE. Support from the Japanese Society for Promotion of Science in also acknowledged by the authors.

\section{References}

Banaszkiewicz, M., H. Fahr, and K. Scherer, Evolution of dust particle orbits under the influence of solar wind outflow asymmetries and the formation of the zodiacal dust cloud, Icarus, 107, 358-374, 1994.

Belton, M. J. S., Dynamics of interplanetary dust, Science, 151(3706), 3544, 1966.

Belton, M. J. S., Dynamics of interplanetary dust particles near the Sun, in The Zodiacal Light and the Interplanetary Medium, NASA SP-150, edited by J. L. Weinberg, pp. 301-306, National Technical Information Service, Springfield, Virginia, 1967.

Grün, E., H. Fechtig, H. A. Zook, and R. H. Giese, Collisional balance of the meteoritic complex, Icarus, 62, 244-272, 1985.

Hoeksema, J. T. and P. H. Scherrer, The solar magnetic field-1976 through 1985, Rept. UAG-94, WDC-A, 1986.

Ishimoto, H., Collisional evolution and the resulting mass distribution of interplanetary dust, Earth Planets Space, 50, this issue, 521-529, 1998.

Isobe, S. and A. S. Kumar, An explanation for time dependent variability of the solar dust ring, Astrophys. Space Sci., 205, 297-303, 1993.

Kimura, H. and I. Mann, The electric charging of interstellar dust in the Solar system and consequences for its dynamics, Astrophys. J., 499, 454-462, $1998 \mathrm{a}$

Kimura, H. and I. Mann, Brightness of the solar F-corona, Earth Planets Space, 50, this issue, 493-499, 1998 b.

Kimura, H., H. Ishimoto, and T. Mukai, A study on solar dust ring formation based on fractal dust models, Astron. Astrophys., 326, 263-270, 1997.
Kimura, H., I. Mann, and T. Mukai, Influence of dust shape and material composition on the solar F-corona, Planet. Space Sci., 1998 (in press).

Krivov, A. V. and D. P. Hamilton, Martian dust belts: Waiting for discovery, Icarus, 128, 335-353, 1997.

MacQueen, R. M., Infrared observations of the outer solar corona, Astrophys. J., 154, 1059-1076, 1968.

Mann, I., The solar $F$-corona-Calculations of the optical and infrared brightness of circumsolar dust, Astron. Astrophys., 261, 329-335, 1992.

Mann, I., Dust near the Sun, in Physics, Chemistry, and Dynamics of Interplanetary Dust (ASP Conf. Series, vol. 104), edited by B. A. S. Gustafson and M. S. Hanner, pp. 315-320, Kluwer, Dordrecht, 1996.

Mann, I. and H. Kimura, Dust near the Sun, in Advances in Dusty Plasmas, edited by P. K. Shukla, D. A. Mendis, and T. Desai, pp. 346-351, World Scientific, Singapore, 1997.

Mann, I., H. Okamoto, T. Mukai, H. Kimura, and Y. Kitada, Fractal aggregate analogues for near solar dust properties, Astron. Astrophys., 291, 1011-1018, 1994.

Mukai, T. and T. Yamamoto, A model of the circumsolar dust cloud, Publ. Astron. Soc. Japan, 31, 585-595, 1979.

Mukai, T., H. Ishimoto, T. Kozasa, J. Blum, and J. M. Greenberg, Radiation pressure forces of fluffy porous grains, Astron. Astrophys., 262, 315-320, 1992.

Peterson, A. W., Experimental detection of thermal radiation from interplanetary dust, Astrophys. J., 148, L37-L39, 1967.

Peterson, A. W., The coronal brightness at 2.23 microns, Astrophys. J., 155 1009-1015, 1969.

Rusk, E. T., The effect of the solar magnetic field on dust-particle orbits in the $F$ corona, Astron. J., 96, 1447-1454, 1988.

Shestakova, L. I. and L. V. Tambovtseva, Dynamics of dust grains near the Sun, Astron. Astrophys. Trans., 8, 59-81, 1995.

van de Hulst, H. C., Zodiacal light in the solar corona. Astrophys. J., 105, 471-488, 1947.

A. Krivov (e-mail: krivov@astro.spbu.ru), I. Mann, and H. Kimura 